\title{
A Novel Utility Sentient Approach for Mining Interesting Association Rules
}

\author{
Shankar.S and T.Purusothaman
}

\begin{abstract}
Utility-based data mining is a new research area that concentrates on all types of utility factors in data mining processes and is targeted at incorporating utility considerations in both predictive and descriptive data mining tasks. Discovering interesting association rules that are utilized to improve the business utility of an enterprise has long been recognized in data mining community. This necessitates identifying interesting association patterns that are both statistically and semantically important to the business utility. Classical association rule mining techniques are capable of identifying interesting association patterns but they have failed to associate the user's objective and utility in mining. In this paper, we have proposed an approach for mining novel interesting association patterns from transaction data items of an enterprise to improve its business utility. The approach mines novel interesting association patterns by providing importance to significance, utility and subjective interestingness of the users. The novel interesting patterns mined using proposed approach can be used to provide valuable suggestions to the enterprise to improve its business.
\end{abstract}

Index Terms-Association Rules, Data Mining, Economic Utility, Frequent Patterns, FP-growth, Significance, Subjective Interestingness.

\section{INTRODUCTION}

Data Mining is a remarkable field of contemporary research and development with regard to computer science that is alluring greater interest from a huge variety of people. The chief motivation for data mining stems from the decision support problems that troubled a majority of business organizations [1,3]. Data mining, also known as Knowledge Discovery in Databases, has been defined as "The nontrivial extraction of implicit, previously unknown, and potentially useful information from data" [2]. Machine learning and various statistical and visualization techniques are utilized by Data Mining in order to ascertain and represent knowledge in an easily interpretable form [4]. Descriptive mining and Predictive mining are the two customary classifications of data mining tasks. The information that is 'mined' is expressed as a model of the semantic structure of the dataset, where in the prediction or classification of the obtained data is facilitated with the aid of the model [5]. Recently,

Mr. Shankar. S, Assistant Professor, Dept. of Information Technology, Sri Krishna College of Engineering \& Technology, Coimbatore, TamilNadu, India. Pin: 641008, Ph: +91-9894379663.

Dr.T.Purusothaman, Assistant Professor, Dept. of Computer Science and Engineering, Government College of Technology, Coimbatore, TamilNadu, India. Pin: 641013, Ph: +91-422-243222 incorporating utility considerations in data mining tasks is gaining popularity in data mining research.

One topic that includes all the perspectives of economic utility in Data Mining and that is destined at assimilating utility considerations for predictive mining tasks and descriptive mining tasks as well. With the computers and e-commerce gaining recognition far and wide, the availability of transactional databases is on an all time high. Association Rule Mining and determining the correlation of the items present in the transaction records are the chief points of focus with regard to Data Mining on transactional databases. Since qualitative properties such as significance, utility etc., are necessary to completely utilize the attributes in the dataset, the researchers from the data mining community are anxious about these qualitative aspects of attributes in comparison to considering the quantitative ones (e.g. number of appearances in a database etc). Discovering interesting association rules, which are used to improve the business utility of an enterprise, has long been recognized in data mining community. This necessitates identifying interesting association patterns that are both statistically and semantically important to the business utility.

Even though several algorithms are available in the literature for association rule mining, [12-20] a good number of them deal with efficient implementations rather than the production of effective rules $[11,16,18]$. The techniques that aid in the extraction of suitable and genuine association patterns are mostly quantitative in nature [10, 12, 13, 17]. In order to completely utilize the attributes of a dataset the qualitative attributes are necessary. In addition, some methods are available in the literature for mining weighted association rules [22, 23]. Incorporating utility in item-set mining has gained popularity in recent years [7, 8]. In our work, we have focused on user's objective and its utility which is not reported in the literate of classical association rule mining techniques. In addition to user's objective and its utility, we have also chosen another parameter, the subjective interestingness. The main goal of our approach is to identify novel and interesting association patterns from the historical buying patterns of an enterprise to ascend its business further. The proposed approach aims to identify interesting patterns that are both statistically and semantically important to improve the business utility.

The rest of the paper is organized as follows: Section 2 presents a brief review of the algorithms available in the literature for association rule mining. The proposed approach for mining novel interesting association patterns is 
discussed in detail in Section 3. The experimental results are presented in Section 4 and conclusions are summed up in Section 5.

\section{RELATED WORK}

A variety of previous works in the field of Association Rule Mining and Utility based Data Mining serve as the inspiration behind the proposed methodology.

Rakesh Agarwal et al. [9] have proposed an efficient algorithm that generates all significant association rules between items in the database. Their algorithm incorporates buffer management and novel estimation and pruning techniques. Ramakrishnan Srikant et al. [10] have introduced the problem of mining association rules in large relational tables containing both quantitative and categorical attributes. They have also introduced a measure of partial completeness which quantifies the information lost due to partitioning.

Charu C. Aggarwal et al. [13] have provided a survey of research on association rule generation. They have discussed a number of variations of the association rule problem which have been proposed in the literature and their practical applications. C.H. Cai et al. [22] have proposed a method of mining weighted association rule. They have provided two different definition of weighted support: without normalization and with normalization and proposed a new algorithm based on the support bounds.

Wei Wang et al. [23] have proposed a two-fold approach, where the frequent item sets were first generated and then the maximum weighted association rules were derived using an "ordered" shrinkage approach. Frans Coenen and Paul Leng [14] have proposed a method for identifying frequent sets, which reduces the task by means of an efficient restructuring of the data accompanied by a partial computation of the totals required.

Liang Dong and Christos Tjortjis [17] have proposed an enhancement with a memory efficient data structure of a quantitative approach to mine association rules from data. They have combined the best features of the three algorithms (the Quantitative Approach, DHP, and Apriori) in their approach. Ferenc Bodon [11] has described an implementation of APRIORI algorithm, which outperformed all implementations available.

Chuan Wang and Christos Tjortjis [18] have proposed an efficient algorithm for mining association rules, which first identifies all large itemsets and then generates association rules. Their approach has reduced large itemset generation time, known to be the most time-consuming step, by scanning the database only once and using logical operations in the process. Verma Keshri et al. [19] have proposed a novel algorithm to find association rule on time dependent data using efficient T-tree and P-tree data structures. The algorithm has elaborated the significant advantage in terms of time and memory while incorporating time dimension.

Yubo Yuan and Tingzhu Huang [20] have proposed an algorithm for efficient generation of large frequent candidate sets, called Matrix Algorithm. The algorithm generated a matrix which entries 1 or 0 by passing over the cruel database only once, and then the frequent candidate sets were obtained from the resulting matrix. Finally association rules were mined from the frequent candidate sets. Girish K. Palshikar et al. [21] have proposed the concept of heavy itemset, which compactly represents an exponential number of rules. They have provided an efficient theoretical characterization of a heavy itemset. They have also presented an efficient greedy algorithm to generate a collection of disjoint heavy itemsets in a given transaction database.

Jieh-Shan Yeh et al. [7] have proposed a novel utility-frequent mining model to identify all itemsets that can generate a user specified utility in transactions, in which the percentage of such transactions in database is not less than a minimum support threshold. They have proposed a bottom-up two-phase algorithm, BU-UFM, for efficiently mining utility-frequent item sets. A top-down two-phase algorithm, TD-UFM, for mining utility-frequent item sets is also presented. Vid Podpe ${ }_{j}$ can et al. [8] have proposed a novel efficient algorithm FUFM (Fast Utility-Frequent Mining) which finds all utility-frequent itemsets within the given utility and support constraints threshold.

\section{Novel InTEResting AsSOciation RUle Mining}

In this section, we have presented our approach for association rule mining. The principal objective of our research is to mine novel interesting association patterns from the transaction data items of an enterprise to improve its business. The transaction data items depict the buying patterns of customers. From the historical buying patterns, our approach aims to mine novel interesting association patterns which are not present in transactions, but are used to ascend the enterprise's business more. In our approach, significance, utility and subjective interestingness of the users are taken into consideration along with the frequency of items. The significance of item is taken into consideration because each item in transactions will have different significance of importance. The utility of an item is important because it contains information about subjectively defined utility such as profit in dollars or some other variety of utility.

The quantity of 'interest' that a pattern evokes upon inspection is captured by the Interestingness measures. Since the Interesting patterns offer ample opportunities for express action which in turn accounts for profitable results, they are considered vital in data mining. Basically, the interestingness of an association pattern is prejudiced. Interestingness plays a vital role for the efficacy of an enterprise as well. Generally, the interestingness measures can be classified into objective and subjective measures. In case of the objective measures the interestingness is expressed with the aid of statistical or mathematical criteria, whereas in subjective measures, more practical criteria such as the suddenness or applicability are considered. 


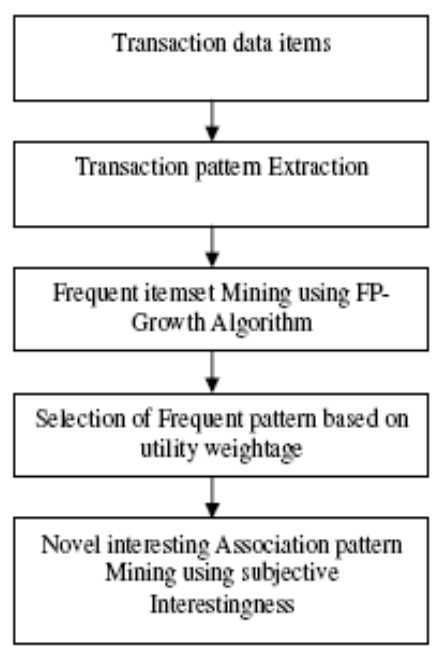

Fig. 1 Block diagram of Proposed Approach

The above figure shows the block diagram of our approach. The major steps involved in our approach are as follows. Initially, the significance weightage of all the items with regard to profit is calculated. The transaction data items are initially scanned for frequent item sets using FP-Growth algorithm. After that, the frequent patterns with utility weightage greater than a threshold value are chosen. As a final point, novel interesting association patterns are mined from the selected patterns based on the subjective interestingness of the users.

\section{A. Association Rule Mining}

This sub-section briefly explains the generalized association rule mining. The buying patterns of customers from the basket data can efficaciously be mined by employing Association rules. Support and confidence measures serve as the basis for customary techniques in association rule mining. The task of mining association rules is defined as follows:

$I S=\left\{i_{1}, i_{2}, i_{3}, \mathrm{~K}, \mathrm{i}_{\mathrm{m}}\right\} \quad$ a $\quad$ set of items and $T D I=\left\{t_{1}, t_{2}, t_{3}, \mathrm{~K}, \mathrm{t}_{\mathrm{n}}\right\}$ be a set of transaction data items, where $t_{i}=\left\{I S_{i 1}, \mathrm{IS}_{\mathrm{i} 2}, \mathrm{IS}_{\mathrm{i} 3}, \mathrm{~K}, \mathrm{IS}_{\mathrm{ip}}\right\}, p<=m$ and $I S_{i j} \in \mathrm{IS}$, if $X \subseteq I$ with $k=|X| \mid$ is called a $k$-item set or simply an item set. An expression, where $X, Y$ are item sets and $X \cap Y=\Phi$ holds is called an association rule $X \Rightarrow Y$.

The measure of number of transactions $\mathrm{T}$ supporting an item set $X$ with respect to TDI is termed as the Support of an item set.

$$
\operatorname{Support}(X)=|\{T \in T D I \mid X \subseteq T\} / T D I|
$$

The ratio of the number of transactions that hold $X \cup \mathrm{Y}$ to the number of transactions that hold $X$ is said to be the confidence of an association rule $X \Rightarrow Y$

$$
\operatorname{Conf}(X \rightarrow Y)=\operatorname{Supp} X \cup Y / \operatorname{Supp}(X)
$$

\section{B. Significance Weightage Calculation}

The first step of our approach is significance weightage calculation. The significance weightage of each item with respect to profit is calculated using the following equation.

$$
S W=P / \sum P_{i}, \mathrm{i}=1 \text { to } \mathrm{n}
$$

Where $n$ is the number of items and $P$ is the profit of an item. Once the weightage is calculated, the data items in the transaction database are represented as a matrix for pattern extraction as follows.

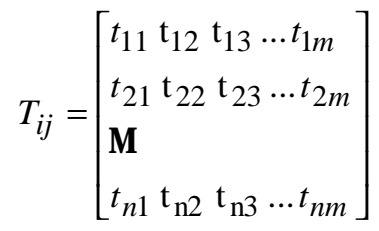

Using the above matrix, the transaction patterns are extracted as a set as follows: where $\Delta_{I}$ represents the item index of all elements in $T_{i j}$.

$T_{i}=\left\{\Delta_{I}\left(\mathrm{t}_{\mathrm{ij}}\right): \mathrm{t}_{\mathrm{ij}} \neq 0\right\}, \mathrm{i}=1, \mathrm{~K}, \mathrm{n}$ and $\mathrm{j}=1, \mathrm{~K}, \mathrm{~m}$ $T_{n}<<\mathrm{T}_{\mathrm{i}}$

The extracted transaction patterns $T_{n}$ are then fed as input to FP-Growth algorithm to find frequent patterns.

\section{FP-Growth Algorithm}

One of the contemporary approaches for frequent item set mining is the FP-growth algorithm [24]. A prefix tree representation of the given database of transactions (called an FP-tree) serves as the basis for the FP-growth algorithm. This can save remarkable amounts of memory for hosting the transactions.

The FP growth algorithm begins with the building of a memory structure called FP-tree. Once the FP-tree is built, the actual FP-growth procedure is recursively applied to it. All the frequent item sets are discovered by this process in a depth-first manner by analyzing projections (conditional FP-trees) of the tree with regard to the frequent prefixes found so far.

\section{Frequent Pattern Selection Based on UtilityWeightage}

In this sub-section, we have presented the selection of frequent patterns based on utility weightage. The frequent patterns extracted using FP-Growth algorithm, are given as input to this stage. The utility weightage of each frequent pattern is calculated using the following equation.

$$
W u=\sum_{i=1}^{n} f * S W_{i}
$$

Where $W u$ represents the utility weightage, $f$ represents the frequency of pattern and $S W_{i}$ represent the significance weightage of $i^{t h}$ item in the current pattern. Then a set of patterns having utility weightage greater than a predefined threshold value $\alpha$ are chosen as follows.

$$
S_{p}=\left\{F_{p}: \mathrm{P}\left(\mathrm{F}_{\mathrm{p}}\right)\right\}
$$




$$
P\left(F_{p}\right)=W_{u}>\alpha
$$

Where $S_{p}$ is the selected patterns set, $\alpha$ is the predefined threshold value for the selection of patterns and $W u$ is the utility weightage.

\section{E. Interesting Association Rule Mining}

The novel interesting association patterns are mined from the selected frequent patterns using the algorithm described in this sub-section. The algorithm implicitly utilized the subjective interestingness of users in interesting pattern mining. The selected frequent patterns from previous sub-section and the output of FP-Growth algorithm are given as input to this algorithm. The algorithm mines novel interesting association patterns, which is not present in transactions, but used to ascend the business. Initially, for every selected pattern, the items are discretely considered and further the patterns with occurrence of each of the item are identified from the frequent patterns. The set difference of the selected item set (pattern) and the identified item set is obtained. The items in the resulting patterns are discretely considered and paired with its reference item and taken into consideration. For every selected pattern, we can get a set of patterns, each with two items. The pseudo code for the above operation is given below.

\section{Assumptions:}

$$
\begin{aligned}
& S p_{i j} \neq \text { Individual item in a selected pattern } \\
& S p_{i} \ddagger \text { A pattern in selected patterns } \\
& F_{p g} \ddagger \text { Frequent itemsets from FP-Growth } \\
& \text { algorithm } \\
& R P \quad \ddagger \text { Resulting Patterns }
\end{aligned}
$$

Pseudo Code:

for each selected pattern

for each item in selected pattern

for each frequent pattern

$$
\begin{aligned}
& \text { If } S p_{i j} \subset F p g_{i} \\
& \quad f p<<F p g_{i} \backslash S p_{i}
\end{aligned} \text {; } \begin{aligned}
& \text { end if } \\
& T P 1<<\mathrm{S}_{\mathrm{p}_{\mathrm{ij}}} \| \mathrm{fp} ;
\end{aligned}
$$

end for

$$
T P 2<<\mathrm{TP} 1
$$

$$
\begin{gathered}
\text { end for } \\
R P<<T P 2 ; \\
\text { end for }
\end{gathered}
$$

From the identified interesting pattern set of a selected pattern, the patterns with equal second item are considered as a group and its weightage is calculated by adding the weightage of patterns present in it. The weightage of a pattern is calculated by the summation of its item's weightages. By multiplying the frequency with the significance weightage, an item's weightage is calculated. The frequency of an item is the count of that item in transactions having both the items in the pattern. The frequency of item $X$ in pattern $X \rightarrow \mathrm{Y}_{\text {is calculated as }}$ follows:

$$
C=\sum_{i=1}^{n} t c(X) \text { Where } t c(X Y) \neq 0
$$

In the above equation $t c(X)$ is the count of item $X$ in current transaction and $\mathrm{n}$ is the total number of transactions. The interestingness weightage of a pattern group is calculated by the following equation.

$$
I w=\sum_{\mathrm{j}=\mathrm{i}}^{\mathrm{m}}\left(\sum_{\mathrm{i}=0}^{1} C_{i} W_{i}\right)
$$

Where $I_{w}$ represents interestingness weightage and $\mathrm{m}$ represents the no of patterns in a group. Afterwards, the second item from each group is combined with its corresponding selected pattern and the interestingness weightage of that group is assigned to the newly formed pattern. The newly formed patterns are sorted based on their interestingness weightage. If more than one pattern occurs with same last item, the pattern with highest interestingness weightage is only considered for further process. The patterns with interestingness weightage greater than a threshold value $\psi$ are chosen. The resulting patterns are the novel interesting association patterns mined using our approach.

\section{EXPERIMENTAL RESULTS}

In this section, we have presented the analysis of our experimental results. The proposed algorithm is implemented in Java.

Table 1 details the profit and weightage of the sample items taken into consideration. A set of sample transactions are shown in table 2. Table 1 and table 2 are fed as inputs to the various algorithms mentioned in section 3.3, 3.4 and 3.5. The frequencies of occurrence of the frequent patterns are tabulated in table 3 . Table 4 shows the utility weightage of each of the patterns. Table 5 contains the patterns with weightage greater than 2.0. The interestingness weightage of the patterns where in the items of the selected pattern occur are tabulated in table 6 . From patterns in table 6 having an identical last item the pattern with the greatest interestingness weightage alone is considered and the rest are dropped. Table 8 shows the patterns further filtered from table 7 with the help of a minimum threshold, 10.0. 
INPUT TABLE-I

WeIGHTAGEITEMS

\begin{tabular}{|l|l|l|l|}
\hline ID & ITEM & $\begin{array}{l}\text { PROFI } \\
\text { T }\end{array}$ & Significance_weightage \\
\hline 1 & $\mathrm{~A}$ & 60 & 0.285714285714286 \\
\hline 2 & $\mathrm{~B}$ & 10 & $4.76190476190476 \mathrm{E}-02$ \\
\hline 3 & $\mathrm{C}$ & 30 & 0.142857142857143 \\
\hline 4 & $\mathrm{D}$ & 90 & 0.428571428571429 \\
\hline 5 & $\mathrm{E}$ & 20 & $9.52380952380952 \mathrm{E}-02$ \\
\hline
\end{tabular}

INPUT TABLE-II

CustomersTransactions

\begin{tabular}{|l|l|l|l|l|l|}
\hline TID & A & B & C & D & E \\
\hline 1 & 10 & 1 & 4 & 1 & 0 \\
\hline 2 & 0 & 1 & 0 & 3 & 0 \\
\hline 3 & 2 & 0 & 0 & 1 & 0 \\
\hline 4 & 0 & 0 & 1 & 0 & 0 \\
\hline 5 & 1 & 2 & 0 & 1 & 3 \\
\hline 6 & 1 & 1 & 1 & 1 & 1 \\
\hline 7 & 0 & 2 & 3 & 0 & 1 \\
\hline 8 & 0 & 0 & 0 & 1 & 2 \\
\hline 9 & 7 & 0 & 1 & 1 & 0 \\
\hline 10 & 0 & 1 & 1 & 1 & 1 \\
\hline
\end{tabular}

TABLE-III

FP-GROWTH

\begin{tabular}{|l|l|}
\hline \multicolumn{1}{|c|}{ Patterns } & Frequent \\
\hline \hline A D & 5 \\
\hline D & 8 \\
\hline A C D & 3 \\
\hline B D & 5 \\
\hline A B D & 3 \\
\hline C D & 4 \\
\hline E D & 4 \\
\hline C B D & 3 \\
\hline E B D & 3 \\
\hline A & 5 \\
\hline A C & 3 \\
\hline A B & 3 \\
\hline C & 6 \\
\hline E C B & 3 \\
\hline C B & 4 \\
\hline E C & 3 \\
\hline E B & 4 \\
\hline E & 5 \\
\hline B & 6 \\
\hline
\end{tabular}

TABLE-IV

FINDING UTILITY WEIGHTAGE

\begin{tabular}{|l|l|l|}
\hline $\begin{array}{l}\text { Pattern } \\
\text { S }\end{array}$ & Frequency & utility_weightage \\
\hline A D & 5 & 3.571428571428571 \\
\hline D & 8 & 3.4285714285714284 \\
\hline A C D & 3 & 2.571428571428571 \\
\hline
\end{tabular}

\begin{tabular}{|l|l|l|}
\hline B D & 5 & 2.380952380952381 \\
\hline A B D & 3 & 2.2857142857142856 \\
\hline C D & 4 & 2.2857142857142856 \\
\hline E D & 4 & 2.095238095238095 \\
\hline C B D & 3 & 1.857142857142857 \\
\hline E B D & 3 & 1.7142857142857142 \\
\hline A & 5 & 1.4285714285714284 \\
\hline A C & 3 & 1.2857142857142856 \\
\hline A B & 3 & 1.0 \\
\hline C & 6 & 0.8571428571428571 \\
\hline E C B & 3 & 0.857142857142857 \\
\hline C B & 4 & 0.7619047619047619 \\
\hline E C & 3 & 0.7142857142857142 \\
\hline E B & 4 & 0.5714285714285714 \\
\hline E & 5 & 0.4761904761904761 \\
& & 6 \\
\hline B & 6 & 0.2857142857142857 \\
\hline
\end{tabular}

TABLE-V

SElECted PATterns From TABLE-4 Where UTILITY_WEIGHTAGE $>2.0$

\begin{tabular}{|l|l|l|}
\hline Patterns & Frequency & utility_weightage \\
\hline A D & 5 & 3.571428571428571 \\
\hline D & 8 & $\begin{array}{l}3.428571428571428 \\
4\end{array}$ \\
\hline A C D & 3 & 2.571428571428571 \\
\hline B D & 5 & 2.380952380952381 \\
\hline A B D & 3 & $\begin{array}{l}2.285714285714285 \\
6\end{array}$ \\
\hline C D & 4 & $\begin{array}{l}2.285714285714285 \\
6\end{array}$ \\
\hline E D & 4 & 2.095238095238095 \\
\hline
\end{tabular}

TABLE-VI

CALCUlating InTERESTINGNESS WEIGHTAGE

\begin{tabular}{|l|l|}
\hline group & $\begin{array}{l}\text { Interestingness } \\
\text { weightage }\end{array}$ \\
\hline C D A & 14.142857142857142 \\
\hline B D A & 11.761904761904761 \\
\hline A B D C & 10.238095238095237 \\
\hline A D C & 8.714285714285714 \\
\hline A C D B & 8.428571428571427 \\
\hline E D A & 8.142857142857142 \\
\hline D A & 8.142857142857142 \\
\hline A D B & 6.904761904761904 \\
\hline C D B & 4.809523809523809 \\
\hline B D C & 4.238095238095238 \\
\hline E D B & 4.142857142857142 \\
\hline E D C & 3.7142857142857144 \\
\hline C D E & 3.380952380952381 \\
\hline A C D E & 3.380952380952381 \\
\hline D B & 3.2857142857142856 \\
\hline A B D E & 3.238095238095238 \\
\hline B D E & 3.238095238095238 \\
\hline D C & 2.7142857142857144 \\
\hline
\end{tabular}




\begin{tabular}{|l||l|}
\hline group & $\begin{array}{l}\text { Interestingness } \\
\text { weightage }\end{array}$ \\
\hline D E & 2.380952380952381 \\
\hline A D E & 2.380952380952381 \\
\hline
\end{tabular}

TABLE-VII

SELECTED PATTERNS FROM TABLE-6

\begin{tabular}{|l|l|}
\hline group & $\begin{array}{l}\text { Interestingness } \\
\text { weightage }\end{array}$ \\
\hline C D A & 14.142857142857142 \\
\hline A B D C & 10.238095238095237 \\
\hline A C D B & 8.428571428571427 \\
\hline C D E & 3.380952380952381 \\
\hline
\end{tabular}

TABLE-VIII

NOVEL INTERESTING PATTERNS

\begin{tabular}{|l|l|}
\hline group & Interestingness_weightage \\
\hline C D A & 14.1428571428571 \\
\hline A B D C & 10.2380952380952 \\
\hline
\end{tabular}

If items $\mathrm{C}, \mathrm{D}$ and $\mathrm{A}$ tend to go together then it is better to place these items side by side. The items such as A which have close proximity will enhance the chances of a customer who comes to buy only C, D buying A as well. For example a person, who comes to buy a Jeans, T. Shirt, may buy a belt, or perfumes or toiletries, if they are placed close by. In short, when similar items are placed adjacently the chances of a customer buying the second item as well are much higher even when he/she had no intention of buying it initially.

\section{CONCLUSION}

Association rules have been widely used to determine customer buying patterns from market basket data. Incorporating utility considerations in association rule mining has gained popularity in recent years. Discovering interesting association rules, used to improve business utility of an enterprise has long been recognized in data mining community. This necessitated the identification of interesting association patterns that are both statistically and semantically important to the business utility. In this paper, we have presented a novel approach for mining interesting association patterns to improve the business utility. The proposed approach focuses on utility, significance and interestingness in the mining of interesting association patterns. The mined interesting association patterns have been used in providing valuable recommendation to the enterprise in intensifying its business utility.

\section{REFERENCES}

[1] S. Tsur, "Data dedging," IEEE Data Engineering Bulletin, 13(4):58-63, December 1990.

[2] Frawley, W., Piatetsky-Shapiro, G., Matheus, C.” Knowledge Discovery in Databases: An Overview". AI Magazine, Fall 1992, pp. 213-228.

[3] J. T-L. Wang, G-W. Chirn, T. G. Marr, B. Shapiro, D. Shasha, and Ii. Zhang, "Combinatorial pattern discovery for scientific data: some preliminary results," In Proceedings of the 1994 ACM SIGMOD International Conference on Management of Data, pages 115-125, Minneapolis, MN, May 24-27 1994.

[4] Soundararajan E., Joseph J.V.M., Jayakumar C. and Somasekharan M., "Knowledge Discovery Tools and Techniques," In Proceedings of the Conference on Recent Advances in Information Technology, IGCAR, pp.141 -145, July 14-15, 2005.

[5] Cunningham, S. J. and Holmes, G., "Developing innovative applications in agriculture using data mining," In the Proceedings of the Southeast Asia Regional Computer Confederation Conference, Singapore, 1999.

[6] Weiss G., Zadrozny B., Saar-Tsechansky M., "Utility-based data mining" 2006 workshop report. SIGKDD Explorations, volume 8, issue 2.

[7] Jieh-Shan Yeh, Yu-Chiang Li, and Chin-Chen Chang, "Two-Phase Algorithms for a Novel Utility-Frequent Mining Model," PAKDD 2007, Lecture Notes in Computer Science, vol. 4819 ,Springer Berlin / Heidelberg, pp. 433-444, 2007.

[8] Vid Podpecan, Nada Lavrac, and Igor Kononenk, "A fast algorithm for mining utility-frequent itemsets," The 11th European Conference on Principles and Practice of Knowledge Discovery in Databases, September 21, 2007.

[9] Agrawal, R., Imielinski, R., Swami, A., 1993, "Mining Associations between Sets of Items in Large Databases", Proceedings of the ACM SIGMOD, Washington, DC, pp.207-216, May 1993.

[10] Srikant, R. and Agrawal, R., "Mining Quantitative Association Rules in Large Relational Tables." In Proc. of ACM SIGMOD Conf. on Management of Data. ACM Press, (1996), 1-12.

[11] Bodon, F.: “A Fast Apriori implementation", In ICDM Workshop on Frequent Itemset Mining Implementations, vol. 90, Melbourne, Florida, USA (2003).

[12] Sotiris Kotsiantis and Dimitris Kanellopoulos, "Association Rules Mining: A Recent Overview," GESTS International Transactions on Computer Science and Engineering, Vol.32 (1), 2006, pp. 71-82.

[13] C. C. Aggrawal and P. S. Yu. "Mining large itemsets for association rules". Bulletin of the IEEE Computer Society Technical Committee on Data Engineering, 21(1): 23-31, March 1998.

[14] F. Coenen and P. Leng. "Optimising Association Rule Algorithms Using Itemset Ordering" Research and Development in Intelligent Systems XVIII: Proc ES2001 Conference, Springer, pages 53-66, 2001.

[15] F. Coenen and P. Leng. "Finding Association Rules with Some Very Frequent Attributes". In Proceedings of PKDD2002, LNAI 2431, pages 99-111, 2002.

[16] Lee, C.-H., Chen, M.-S., Lin, C.-R. , "Progressive Partition Miner: An Efficient Algorithm for Mining General Temporal Association Rules," IEEE Transactions on Knowledge and Data Engineering, Vol. 15, No. 4, (2003), 1004 - 1017.

[17] L. Dong and C. Tjortjis, "Experiences of Using a Quantitative Approach for Mining Association Rules," Lecture Notes in Computer Science Series, Vol. 2690, Springer-Verlag, (2003), 693-700.

[18] Wang, C., Tjortjis, C., "PRICES: An Efficient Algorithm for Mining Association Rules," Lecture Notes in Computer Science, Volume 3177, Jan 2004, Pages 352 - 358 .

[19] Verma, K., Vyas, O.P., Vyas, R., "Temporal Approach to Association Rule Mining Using T-Tree and P-Tree", Lecture Notes in Computer Science, Volume 3587, Jul 2005, Pages 651 - 659.

[20] Yuan, Y., Huang, T., "A Matrix Algorithm for Mining Association Rules", Lecture Notes in Computer Science, Volume 3644, Sep 2005, Pages 370 - 379 .

[21] GK Palshikar, MS Kale, MM Apte, "Association rules mining using heavy itemsets", Data and Knowledge Engineering, Vol. 61, No. 1, pp. 93-113, 2007.

[22] Cai, C.H., Fu, A.W-C., Cheng, C. H., Kwong, W.W. "Mining Association Rules with Weighted Items". In: Proceedings of 1998 Intl. Database Engineering and Applications Symposium (IDEAS'98), pages 68--77, Cardiff, Wales, UK, July 1998

[23] Wang, W., Yang, J., Yu, P. S. "Efficient Mining of Weighted Association Rules (WAR)". In: Proceedings of the KDD, Boston, MA, August 2000, pp. 270-274

[24] Han, J., Pei, J., and Yin, Y., "Mining frequent patterns without candidate generation," In Proceedings of 2000 ACMSIGMOD International Conference on Management of Data (SIGMOD’00), pp. 1-12, Dallas, TX, 2000. 
Mr. Shankar. S is currently working as Assistant Professor in the Department of Information Technology at Sri Krishna College of Engineering \& Technology, Coimbatore. He is now doing his Ph.D in Computer Science and Engineering at Anna University, Coimbatore. He did his M.E (CSE) at Kumaraguru College of Technology, Coimbatore and B.E (CSE) at Sri Ramakrishna Engineering College, Coimbatore under Anna University, Chennai and Bharathiar University respectively. He has eight years of teaching experience. He is an IBM certified DB2 professional and has obtained Brain Bench certification in various disciplines. He has presented a number of papers in various National and International conferences. Two of his papers were published in IEEE Explore. He has guided a number of research-oriented as well as application oriented projects organized by well known companies like IBM. He has to his credit a journal publication in IJSCA (International Journal of Soft Computing Applications). His research interests include Data Mining, Soft Computing and Database Management Systems.

The Co-Author Dr.T.Purusothaman is currently working as Assistant Professor (RD) in the department of Computer Science and Engineering and Information technology, Government College of Technology, Coimbatore. He has twenty years of teaching experience. He has completed Ph.D in the area of Network Security and Grid Computing. In his thesis, a novel key management scheme was proposed to provide service only for the paid customers in Internet. He has successfully completed a project funded by DIT (Government of India) in the area of cryptanalysis in the year 2006. He has presented a number of papers in various National and International conferences. Many of his papers were published in IEEE Explore. He has to his credit several International Journal Publications in reputed journals including Journal of Grid Computing, Springer. His research interests include Network Security, Grid Computing and Data Mining. 